\title{
Seroepidemiology of CCHF in Domestic Animals in Endemic Areas in Albania
}

\author{
Përparim Kadriaj ${ }^{*}$ \\ Majlinda Dhimolea-Kota ${ }^{1}$ \\ Enkelejda Velo ${ }^{1}$ \\ Kujtim Mersini ${ }^{2}$ \\ Artan Simaku ${ }^{1}$ \\ Kristaq Berxholi ${ }^{3}$ \\ Silva Bino ${ }^{1}$ \\ ${ }^{1}$ Infectious Disease Control Department, \\ Institute of Public Health, Tirana., Albania; \\ ${ }^{2}$ National Veterinary Epidemiology Unit, \\ Institute of Food Safety ond Veterinary, Tirana, Albania; \\ ${ }^{3}$ Faculty of Veterinary Medicine, \\ Agriculture University, Tirana, Albania; \\ *Correspondent Author
}

Doi: 10.2478/ajis-2018-0043

\begin{abstract}
Crimean-Congo hemorrhagic fever (CCHF) is an arboviral zoonotic infection wich is endemic in some areas of the country. The aim of this study was to assess the seroprevalence of CCHFV in previous and recent endemic areas of the country. This crosssectional serologic study was conducted in period 20102014 by the Institute of Public health in Tirana, Albania. The survey included 152 sera specimen of cattle which were randomly collected from seven districts in Albania over the period 2010-2014. All samples were collected randomly from seven districts in Albania and were examined for anti-CCHFV IgG. Of the total of 152 samples examined, 102 (67.1\%) were positive to IgG ELISA. The highest positivity rate was among cows (88.3\%) and in recent endemic areas of Has and Kukes, $89.7 \%$ and $82.9 \%$ respectively. In recent endemic areas humans can potentially contract the disease as compared to other areas of the country.
\end{abstract}

Keywords: Crimean-Congo hemorrhagic fever, domestic animals, seroprevalence

\section{Introduction}

Crimean-Congo haemorrhagic fever (CCHF) is the most widespread, tick-borne viral disease affecting humans. The disease is endemic in many regions, such as Africa, Asia, Eastern Europe, and the Middle East. The known distribution of CCHFV covers the greatest geographic range of any tick-borne virus and there are reports of viral isolation and/or disease from more than 30 countries across four regions: Africa (Democratic Republic of Congo, Uganda, Mauritania, Nigeria, South Africa, Senegal, Sudan), Asia (China, Kazakhstan, Tajikistan, Uzbekistan, Afghanistan, Pakistan, 
India), Europe (Russia, Bulgaria, Kosovo, Turkey, Greece, Spain), and the Middle East (Iraq, Iran, Kuwait, Saudi Arabia, Oman, United Arab Emirates (UAE)) (1). The geographic distribution of CCHF coincides with that of ixodid ticks, particularly those of the genus Hyalomma. In Europe, Hyalomma marginatum is the main CCHFV vector, while Hyalomma asiaticum appears to be the principal vector in Asia. In 2006, H. marginatum was detected for the first time in the Netherlands and in southern Germany $(2,3)$. Ixodid ticks, especially those of the genus Hyalomma, are both a reservoir and a vector for the virus. The CCHF virus (CCHFV), a Nairovirus of the family Bunyaviridae, has been isolated from 30 species of ixodid tick (3). Numerous domestic and wild animals, such as cattle, goats, sheep, small mammals, rodents, and birds, in which the infection is mainly asymptomatic, serve as amplifying hosts for the virus (4). There is no specific treatment or vaccine against CCHF and it is considered an emerging arboviral zoonotic disease in many countries, possibly due to increased vector bionomics and climate change. Recently, the incidence of CCHF has increased rapidly in the countries of the World Health Organization Eastern Mediterranean Region (WHO EMR), with sporadic human cases and outbreaks of CCHF being reported from a number of countries in the region. Despite the rapidly growing incidence of the disease, there are currently no accurate data on the burden of the disease in the region due to the different surveillance systems for CCHF used in these countries. Moreover, there is no definitive preventive and control strategy for CCHF owing to the fact that many aspects of the disease, such as the maintenance and transmission of the virus and the pathogenesis of the disease in humans, remain poorly understood (5). Considering the zoonotic nature and public health importance the aim of this study was to assess the seroprevalence of CCHFV in previous and recent endemic areas of the country.

\section{Material and Methods}

Blood specimen collection: The survey included 152 sera specimen of cattle which were randomly collected from seven districts in Albania over the period 2010-2014. These localities include (Berat, Elbasan, Gjirokaster, Gramsh, Has, Kukes, Skrapar).

Sterile vacutainers were used to draw the blood from jugular veins and were left to clot. Sera were separated afterwards and stored at $-20^{\circ} \mathrm{C}$ until used.

Laboratory examination: Adaptation of a commercial CCHFV ELISA and IFA.

ELISA commercial test for identifying of specific CCHFV antibodies in human sera (Vector Best, Novosibirsk, Russia) was adapted for testing sera from sheep and goats. All washing steps were performed with PBS-Tween20. Sera were diluted 1:100 in dilution buffer (90\% SDB- and $10 \%$ SPSD-buffer of the manufacturer). Diluted sera (100 I/well) were incubated for $1 \mathrm{~h}$ at $37^{\circ} \mathrm{C}$. After washing the plates, 100 Il/well of rabbit-anti-sheep-lgG-HRP conjugate (Southern Biotech, Birmingham, AL, USA) or rabbit-anti-goat-IgGHRP conjugate (Southern Biotech) were added. Both were diluted 1:6000 in conjugate dilution buffer (by manufacturer) and incubated for $30 \mathrm{~min}$ at $37^{\circ} \mathrm{C}$. After washing the plates, 100 II tetramethylbenzidine (TMB, Bio-Rad, Munich, Germany) solution was added per well, and the reaction was stopped with $\mathrm{H}_{2} \mathrm{SO}_{4}$ after 10 min. The extinction was measured at $\mathrm{k}=450 \mathrm{~nm}$ (reference wavelength $620 \mathrm{~nm}$ ). Serum samples with OD-values (background reaction subtracted) of 0.5 and lower were defined as 'negative', whereas serum samples with OD-values of 0.6 and 0.7 were defined as 'inconclusive' and serum samples with ODvalues of 0.8 and higher were defined as 'positive'. (6)

Statistical analysis: data were analyzed using SPSS software. Chi-square test was used to test the differences between proportions. Statistical significance was set for $p$ value $\leq 0.05$.

\section{Results \& Discussion}

Distribution of domestic animals by district is shown in table 1. As noted, most of domestic animals belong to district of Has (38.2\%) followed by district of Kukes $(27 \%)$ which are recent endemic areas, with a significant difference as compared to other district $(p<0.01)$.

Cows account for $50.7 \%$ of animals, followed by sheep (29.6), bovine (17.1\%) and goats $(2.6 \%),(p<0.01)$. 
Table 1. Distribution of domestic animals by district

\begin{tabular}{|l|c|c|c|c|c|}
\hline \multirow{2}{*}{ District } & \multicolumn{4}{|c|}{ Type of domestic animals } & \multirow{2}{*}{ Total n (\%) } \\
\cline { 2 - 5 } & Bovine & Cow & Goat & Sheep & \\
\hline Berat & 0 & 1 & 0 & 13 & $14(9.2 \%)$ \\
\hline Elbasan & 3 & 0 & 0 & 0 & $3(2.0 \%)$ \\
\hline Gjirokaster & 5 & 0 & 0 & 13 & $18(11.8 \%)$ \\
\hline Gramsh & 7 & 0 & 0 & 0 & $7(4.6 \%)$ \\
\hline Has & 0 & 58 & 0 & 0 & $58(38.2 \%)$ \\
\hline Kukes & 0 & 18 & 4 & 19 & $41(27.0 \%)$ \\
\hline Skrapar & 11 & 0 & 0 & 0 & $11(7.2 \%)$ \\
\hline Total n $(\%)$ & $26(17.1 \%)$ & $77(50.7 \%)$ & $4(2.6 \%)$ & $45(29.6 \%)$ & 152 \\
\hline
\end{tabular}

The serologic results are shown in table 2.

According to ELISA serology the majority of specimens $(67.1 \%)$ resulted positive for IgG to CCHF $[95 \% \mathrm{Cl} 59.3-74.1]$. A very small number of specimens $(3.3 \%)$ resulted cut-off.

Table 2. ELISA IgG serologic results

\begin{tabular}{|l|c|c|c|}
\hline ELISA IgG & $\mathrm{N}$ & $\%$ & $95 \% \mathrm{Cl}$ \\
\hline Cut-off & 5 & $3.3 \%$ & $1.42-7.48$ \\
\hline Negative & 45 & $29.6 \%$ & $22.9-37.3$ \\
\hline Positive & 102 & $67.1 \%$ & $59.3-74.1$ \\
\hline Total & 152 & $100.0 \%$ & \\
\hline
\end{tabular}

Table 3 presents the serologic result by type of animal. The highest positivity rate was among cows $(88.3 \%)$, followed by sheep $(55.6 \%)$, bovine $(30.8 \%)$ and only one case among goats $(25 \%)$, $\mathrm{p}<0.01$.

Table 3. Serologic result by type of animal

\begin{tabular}{|l|c|c|c|c|c|}
\hline Domestic animals & Total specimens & Cut-off & Negative & Positive & Prevalence (\%) of positive specimens \\
\hline Bovine & 26 & 0 & 18 & 8 & $8(30.8 \%)$ \\
\hline Cow & 77 & 3 & 6 & 68 & $68(88.3 \%)$ \\
\hline Goat & 4 & 0 & 3 & 1 & $1(25 \%)$ \\
\hline Sheep & 45 & 2 & 18 & 25 & $25(55.6 \%)$ \\
\hline
\end{tabular}

Table 4. Serologic result by district

\begin{tabular}{|l|c|c|c|c|}
\hline District & Total specimens & Cut-off & Negative & Prevalence (\%) of positive specimens \\
\hline Berat & 14 & 1 & 5 & $8(57.1 \%)$ \\
\hline Elbasan & 3 & 0 & 3 & 0 \\
\hline Gjirokaster & 18 & 0 & 17 & $1(5.6 \%)$ \\
\hline Gramsh & 7 & 0 & 4 & $3(42.9 \%)$ \\
\hline Has & 58 & 3 & 3 & $52(89.7 \%)$ \\
\hline Kukes & 41 & 1 & 6 & $34(82.9 \%)$ \\
\hline Skrapar & 11 & 0 & 7 & $4(36.4 \%)$ \\
\hline Total & 152 & $5(3.3 \%)$ & $45(29.6 \%)$ & $102(67.1 \%)$ \\
\hline
\end{tabular}

As noted from the above table the recent endemic areas of Has and Kukes show the highest positivity rate, $89.7 \%$ and $82.9 \%$ respectively, with a significant difference to other districts $(p<0.01)$. Crimean-Congo hemorrhagic fever (CCHF) is a well-known infectious nosology in Albania since its first report in 1974; however, CCHF primary serological studies have been commenced since 1969. For this cross sectional study, we have tested the blood samples of previous and recent endemic areas of the country. 
From our results we have different values in different areas. We found the presence of infection (antibodies) in animals in areas where cases with hemorrhagic fever were observed more than one to two decades ago, where since then has not been observed cases with hemorrhagic fever in humans. This phenomenon is observed in districts other than Has and Kukes. This indicates that the infection in these areas is still present and we think it can become active. We think that these results should be a signal especially for human service which should take strong observation in these areas and in equivocal cases should immediately take appropriate measures. The results of our study are similar with other studies conducted in Albania regarding the seroprevalence of CCHFV in cattle $(6,7,8)$. In Kosovo the presence of the CCHF antibodies was found in $14 \%$ of livestock, and in $32.6 \%$ of sheep (9).

Countries like Turkey and other Balkan countries with the exception of Greece have recorded circulation of CCHF strains among animal hosts, ticks, and humans, and have established CCHF endemicity (10). The wide outbreaks that occurred in Turkey were preceded by several decades of serologic evidence of a zoonotic CCHF focus. Livestock originated from endemic zones resulted $14 \%$ seropositive on CCHF virus, only sheep were $32.6 \%$ seropositive (11).

The establishment and maintenance of a CCHF endemic focus requires an environment favoring an efficient contact between competent ticks and animal hosts with relatively high prevalence of infection. Heterogeneity in infection levels may occur even within endemic foci, as a result of variable climate and environmental suitability for ticks and animal hosts

Over recent decades, alterations in natural ecosystems, intensive agriculture, global warming, and the exponential increase of movement of people for any cause have provided the framework for the expansion of several vector-borne diseases globally, from endemic areas to neighboring nonendemic areas as well as to distant continents. In animal that were infected the virus was amplified to a high level which was sufficiently enough to transmit to the tick vectors, as shown in former investigational surveys which employed cattle inoculation. Seroconversion was detected among infected cattle due to development of a viremia of a low-tire (12).

In the phase of viremia cattle shed the virus and vulnerable humans can contract the disease through bites of infected ticks (13). The outcome of these studies highlight the significant contribution of cattle in the transmission of the infection.

This finding suggested that cattle may play an important role in the epidemiology of the disease. On the contrary, a high rate of lethality from CCHFV infection is observed among humans which reaches up to $30 \%$.

\section{Conclusions}

The results of this survey suggest that in recent endemic areas humans can potentially contract the disease as compared to other areas of the country. Occupational exposure risk should be reduced among professionals handling with animals by implementing the measures of protection and avoiding risky behaviors (14). Doctors and other medical staff ought to think about this infection in individuals with signs and symptoms similar to CCHFV. Mapping of ticks population in the country and promotion are of paramount importance to prevent future CCHF outbreaks.

\section{References}

World Health Organization. (2013). Crimean-Congo haemorrhagic fever (CCHF) in Pakistan. [Online] Available: http://www.emro.who.int/pandemic-epidemic-diseases/news/cchf-pakistan-june-2013.html (June 14, 2013).

Maltezou, H. C., \& Papa, A. (2010). Crimean-Congo hemorrhagic fever: risk for emergence of new endemic foci in Europe? Travel Med Infect Dis, 8, 139-143.

Kampen, H., Poltz, W., Hartelt, K., Wölfel, R., \& Faulde, M. (2007). Detection of a questing Hyalomma marginatum marginatum adult female (Acari, Ixodidae) in southern Germany. Exp Appl Acarol, 43, 227231. 
Al-Abri, S. S., Al Abaidani, I., Fazlalipour, M., Mostafavi, E., Leblebicioglu, H., ... \& Nguyen, T. M. N. (2017). Current status of Crimean-Congo haemorrhagic fever in the World Health Organization Eastern Mediterranean Region: issues, challenges, and future directions. Int J Infect Dis, 58, 82-89.

Messina, J. P., Pigott, D. M., Golding, N., Duda, K. A., Brownstein, J. S., Weiss, D. J., \& Nuttall, P. A. (2015). The global distribution of Crimean-Congo hemorrhagic fever. Trans R Soc Trop Med Hyg, 109, 503-513.

Lugaj, A., Koni, M., Mertens, M., Groschup, M. H., \& Bërxholi, K. (2014a). Serological survey of Crimean-Congo hemorrhagic fever virus in cattle in Berat and Kolonje, Albania. Albanian J Agric Sci, 13, 325-328.

Lugaj, A., Koni, M., Schuster, I., Mertens, M., Groschup, M. H., \& Berxholi, K. (2014b). A seroepidemiological survey of Crimean-Congo hemorrhagic fever virus among goats and sheep in Lezhe-Torovica Province, Albania. Albanian J Agric Sci, 13, 28-31.

Lugaj, A., Mertens, M., Groschup, M. H., \& Bërxholi, K. (2014c). Serological survey of CCHFV in cattle in 10 regions of Albania. Int J Res Appl Nat Soc Sci, 2, 55-60.

Humolli, I., Dedushaj, I., Zupanac, T. A., \& Muçaj, S. (2010). Epidemiological, serological and herd immunity of Crimean-Congo haemorrhagic fever in Kosovo. Med Arh, 64, 91-93.

Dowall, S. D., Richards, K. S., Graham, V. A., Chamberlain, J., \& Hewson, R. (2012). Development of an indirect ELISA method for the parallel measurement of IgG and IgM antibodies against Crimean-Congo haemorrhagic fever (CCHF) virus using recombinant nucleoprotein as antigen. J Virol Methods, 179, 335341.

Leblebicioglu, H., Ozaras, R., Irmak, H., \& Sencan, I. (2016). Crimean-Congo hemorrhagic fever in Turkey: current status and future challenges. Antiviral Res, 126, 21-34.

Mohamed, M., Said, A., Murad, M., \& Graham, R. (2008). A serological survey of Crimean Congo hemorrhagic fever in animals in the Sharkia Governorate of Egypt. Vet Ital, 44, 513-517.

Swanepoel, R., Shepherd, A. J., Leman, P. A., Shepherd, S. P., \& Miller, G. B. (1985). A common-source outbreak of Crimean-Congo haemorrhagic fever on a dairy farm. S Afr Med J, 68, 635-637.

Abu Salma, A. A. (1995). Meat hygiene in the Sudan: Public health implications of edible offals. MVSc thesis: Faculty of Veterinary Medicine, University of Khartoum, Sudan. 